\title{
Short-term outcomes of CyberKnife therapy for advanced high-risk tumors: A report of 160 cases
}

\author{
YI-SHAN WANG, YUAN-YUAN WANG, PENG JIANG, JIAN-JUN MA, \\ ZHEN QU, XI-LIN WANG, JUN-TI LI and XI-FENG JIA
}

Center for Tumor Treatment, The People's Liberation Army 107th Hospital, Shandong 264002, P.R. China

Received October 6, 2011; Accepted December 1, 2011

DOI: $10.3892 /$ etm.2012.451

\begin{abstract}
The objective of the present study was to evaluate short-term outcomes of CyberKnife therapy in patients with advanced high-risk tumors. A total of 201 target areas from 341 advanced high-risk tumor lesions in 160 patients were treated with CyberKnife. A prescribed dose of 18-60 Gy to the gross tumor volume was delivered in 1-6 fractions to complete the entire treatment in 1 week. Radiographic studies and clinical examinations were performed at 1- to 3-month follow-up intervals, and the results were compared to outcomes of 160 similar advanced high-risk tumor patients who were treated by conformal radiotherapy (CRT). After CyberKnife therapy, the short-term improvement in the quality of life was significant according to radiographic study, radioimmunoassay and ZPS scores of these patients. The total rates of objective efficacy and alleviation of ascities were as high as 66.88 and $67.90 \%$. The short-term outcomes in our series of patients with advanced high-risk tumors treated with CyberKnife appeared to be better compared to conventional CRT. CyberKnife may be an option for patients with incurable advanced high-risk tumors, although further studies of the long-term outcomes are required to confirm the validity.
\end{abstract}

\section{Introduction}

Most advanced high-risk tumors are contraindicated for conventional treatments, such as surgery, chemotherapy and radiotherapy, due to the following characteristics: post-operative recurrence and metastasis (1); significant functional central organ invasion (2); local lymph node nerve cell infiltration or multiple metastases (3-5). On this occasion, symptomatic palliative therapy is preferable in clinical treatment (6). Due to direct invasion, tumors oppress neighboring tissues; thus,

Correspondence to: Dr Yi-Shan Wang, Center for Tumor Treatment, The People's Liberation Army 107th Hospital, 7 Southeastern Street of Zhichu, Yantai, Shandong 264002, P.R. China

E-mail: wangyishan288@163.com

Key words: high-risk tumor, CyberKnife, stereotactic radiotherapy, short-term outcomes the accompanying tumor oppression syndrome leads to great patient suffering (7-9).

In order to improve therapeutic efficacy for advanced high-risk tumors, we tried to eliminate the local lesion by CyberKnife, which was combined with hyperthermia therapy. In the present study, a retrospective evaluation of short-term outcomes of patients treated consecutively with CyberKnife was performed to demonstrate the clinical applicability of CyberKnife for incurable advanced high-risk tumors. Through CyberKnife therapy, we controlled the development of tumors, alleviated the clinical symptoms of patients and reduced adverse reactions.

\section{Patients and methods}

Patient characteristics. One hundred and sixty patients with 341 tumor lesions were recruited; all of them were patients with advanced high-risk tumors who came to the Center for Tumor Treatment, The People's Liberation Army 107th Hospital, for palliative treatment between November 2010 and June 2011. The inclusion criteria were: i) pathological or radiographic confirmation of stage III-IV high-risk tumors; ii) performance status (PS) of 1-4 according to the Eastern Cooperative Oncology Group (ECOG) scale; iii) all the inclusive patients provided informed consent for detection and treatment.

One hundred and one patients were male and 59 were female. All patients were from 6 to 81 years of age, with a mean age of 53.7. The locations of tumors were 55 head and neck cases, 54 chest cases, 34 abdomen cases and 17 vertebral cases. Included were a total of 341 lesions and 201 target area plans among all patients. The number of lesions for each patient ranged from 1 to 24 . The largest tumor lesion was $15 \times 9.5 \times 18 \mathrm{~cm}$, while the smallest was $1 \times 0.5 \times 0.5 \mathrm{~cm}$. Twenty-eight cases were combined with moderate to severe hydrothorax or ascites, and 49 cases had cerebral edema of different degrees.

Methods. CT scans (1.25-mm) were conducted soon after the patients were hospitalized. MRI, PET-CT or DSA image fusion was performed when necessary. For the organs which usually make large respiratory amplitude, 1-3 gold markers were placed into the lung, liver and pancreas, to definitely mark the target areas. We then outlined the target areas and determined the treatment plan and therapeutic doses according to the number, volume, location and stage of the tumor, which 
were usually delivered in 1-6 fractions, and the entire treatment was completed in 1 week. The whole doses ranged from 18 to $60 \mathrm{~Gy}$, with a fractional dose of 4-18 Gy. For patients with somatic tumors, we subjoined radiofrequency ablation diathermy of 2 fractions per week after the CyberKnife therapy, with an equivalent hot dose of $40^{\circ} \mathrm{C} / \mathrm{min}$.

Outcome evaluation and statistical analyses. The outcomes were evaluated based on radiographic examination, radioimmunoassay, ZPS (Zubrod-ECOG-WHO) scores, changes in ascities and the Objective Efficacy Evaluation Criteria. Comparisons between short-term outcomes of CyberKnife therapy and conformal radiotherapy (CRT) were made. Chi-square test was used for the comparison of the rate between the two samples. Differences were considered statistically significant with a P-value $<0.05$. SPSS 13.0 software was used for statistical analysis.

\section{Results}

Radiographic observation. Radiographic study showed tumor shrinkage of different degrees and even disappearance after CyberKnife therapy (18 disappeared, 99 exhibited shrinkage, 35 were unchanged and 8 were enlarged). The recent curative effect of CyberKnife therapy in the radiographic study was $73.13 \%$, and was obviously superior to CRT (3 disappeared, 65 exhibited shrinkage, 70 were unchanged and 22 were enlarged) according to the statistical analysis.

Radioimmunoassay (RIA). In 40 patients, the tumor marker reversed trend and was positively heightened. After CyberKnife therapy, 5 cases became negative, 15 cases decreased to different degrees and 17 cases were unchanged, while 3 cases became more positive.

ZPS scores before and after treatment. After CyberKnife therapy, the ZPS scores of all patients decreased, accompanied by alleviation of tumor oppression syndrome and apparent improvement of the quality of life. The comparison of ZPS scores before and after CyberKnife therapy was performed using the Wilcoxon's test $(\mathrm{P}<0.05)$. Apparent improvement of ZPS scores occurred after CyberKnife therapy.

Changes in ascities. There were 28 patients who presented with different degrees of ascites among the 160 cases. Regarding the alleviation of ascites, CyberKnife treatment was significantly effective in 8 cases (ascites disappeared completely), effective in 11 cases ( $>60 \%$ ascites disappeared after treatment and no new complication appeared), while CyberKnife treatment was ineffective in 9 cases. The total efficacy rate was $67.9 \%$.

Objective efficacy assessment. For patients with advanced high-risk tumors who received repeated treatment, there was still high palliative efficacy after CyberKnife therapy. What should be emphasized is that, although there were no significant changes in radiography, these patients experienced complete improvement in the quality of life after CyberKnife therapy. According to the statistical analysis, CyberKnife therapy is superior to CRT in the aspect of objective effect assessment.

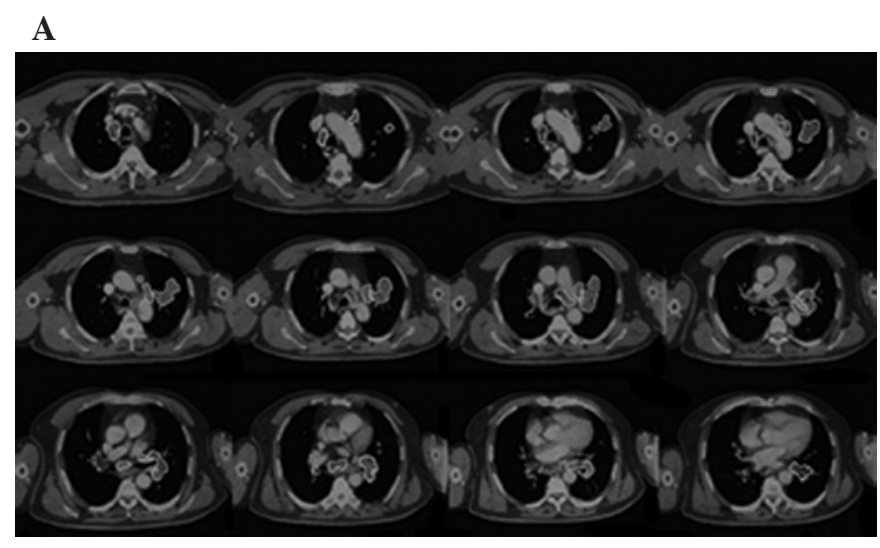

B

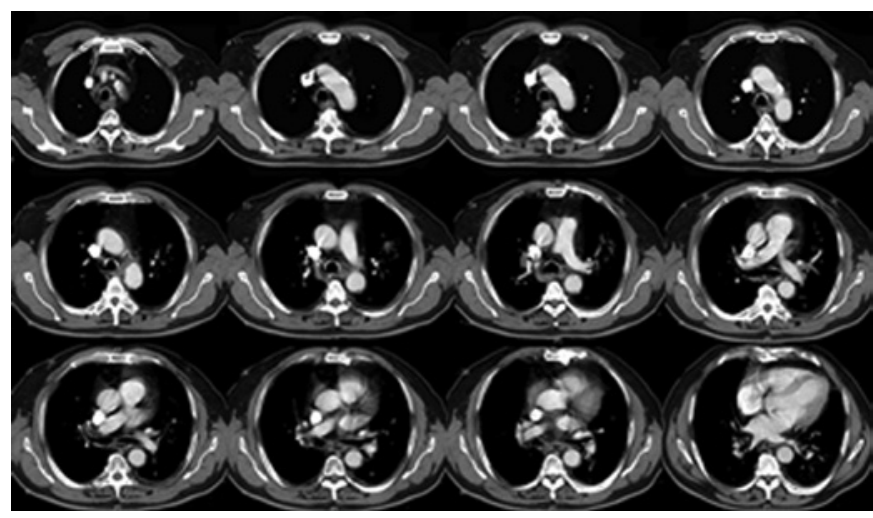

Figure 1. Lung cancer with mediastinal lymph node metastases (15 lesions). CT revealed that tumor lesions completely disappeared after CyberKnife therapy. (A) CT before CyberKnife therapy (2011-01-28); (B) CT after CyberKnife therapy (2011-03-11).

\section{Discussion}

With substantial improvements in radiotherapy technology, stereotactic body radiotherapy, which allows dose-intensification, has led to promising treatment outcomes (10). Cyberknife, the robotic stereotactic radiosurgery system, which is able to precisely deliver a high dose of radiation using an excellent synthesis of modern image-guided localization and a compact robotically positioned linear accelerator, has unique advantages that make it dramatically suited for stereotactic radiotherapy of advanced high-risk tumors $(11,13)$.

However, due to the realization of hypofractions, larger doses and shorter treatment courses when using CyberKnife therapy, numerous studies have consequently placed too much emphasis on the lack of need for patient hospitalization, the short treatment course and non-toxic side effects (12-14). We believe that CyberKnife, due to its precision and flexibility, should be highly individualized, and the blind pursuit of a rapid treatment course and early discharge from the hospital should be avoided (15). During the present study, we found it was extremely necessary to prevent an early tumor-dissolving response and abnormal bilirubin metabolism due to the rapid, short-course killing of tumor cells. The study also discovered that patient neurophil levels suddenly decreased 2 weeks after CyberKnife therapy. If this had not been discovered in time and treated efficiently, there would have been serious consequences for the patients. Therefore, it is necessary for patients with multiple or large tumors to be hospitalized. 
Using a combination of CyberKnife with a variety of imaging techniques, such as CT, MRI, PET-CT and DSA, an actual three-dimensional image of tumors or vascular lesions can be obtained. This provides a good foundation for target area sketching $(16,17)$. However, to date, most experts still refer to the traditional radiobiology model (18); a new biological model has yet to be established. Because of this delay, in China, no uniform treatment exists even for the same disease (19-21). Based on the dosage plan, which was designed according to the linear quadratic model, with the $\alpha / \beta$ value of early response tissues set as 10 , late response tissue as 3 and conversed by conventional $2 \mathrm{~Gy}$ fractionated radiation, we arrived at a dosage which was close to the clinical experienced dose. Yet, a difference with both the clinical practice and foreign reports was noted $(22,23)$. As a more precise therapy modality, CyberKnife realizes hypofracted steretactic treatment and abandons the strict limitation of taking biological effect into consideration, which is different from ordinary radiotherapy. (24-27).

We conclude that CyberKnife is undoubtedly a safer treatment modality for high-risk or highly difficult cases. One patient in our study who had lung cancer with mediastinal lymph node metastases (15 lesions) was definitely a highly difficulty case. After treatment using CyberKnife, the tumor lesions completely disappeared (Fig. 1). After the applied research of CyberKnife in 160 advanced high-risk (or difficult) tumor cases, we believe that CyberKnife cannot only quickly improve the clinical symptoms of patients and obtain satisfactory shortterm curative effects, but it is also suitable for the treatment of high-risk tumors, thus curing the incurable tumors.

\section{Acknowledgements}

This study was partially supported by grants from the National Special Issue of the Ministry of Health of China (No. 2009BX042).

\section{References}

1. Chang BK and Timmerman RD: Stereotactic body radiation therapy: a comprehensive review. Am J Clin Oncol 30: 637-644, 2007.

2. Shirato H, Shimizu S, Kitamura K and Onimaru R: Organ motion in image-guided radiotherapy: lessons from real-time tumor tracking radiotherapy. Int J Clin Oncol 12: 8-16, 2007.

3. Dewas S, Dewas-Vautravers C, Servent V, et al: Results and special considerations when treating elderly patients with CyberKnife: a review of 345 cases. Crit Rev Oncol Hematol 76: 1-7, 2010.

4. Brown WT, Wu X, Wen BC, et al: Early results of CyberKnife image-guided robotic stereotactic radiosurgery for treatment of lung tumors. Comput Aided Surg 12: 253-261, 2007.

5. Bucholz RD, Laycock KA and Cuff LE: CyberKnife stereotactic radiosurgery for intracranial neoplasms, with a focus on malignant tumors. Technol Cancer Res Treat 9: 541-550, 2010.

6. Nagata Y, Matsuo Y, Takayama K, et al: Current status of stereotactic body radiotherapy for lung cancer. Int J Clin Oncol 12: 3-7, 2007.

7. Yamazaki H, Shiomi H, Tsubokura T, et al: Quantitative assessment of inter-observer variability in target volume delineation on stereotactic radiotherapy treatment for pituitary adenoma and meningioma optic tract. Radiat Oncol 6: 10, 2011.
8. Hasney CP, Swanton RG and Friendlander PL: CyberKnife stereotactic radiosurgery for recurrent squamous cell carcinoma of the head and neck following salvage surgery with close or positive margins. Laryngoscope 4: 152, 2010.

9. Oermann EK, Slack RS, Hanscom HN, et al: A pilot study of intensity modulated radiation therapy with hypofractionated stereotactic body radiation therapy (SBRT) boost in the treatment of intermediate to high-risk prostate cancer. Technol Cancer Res Treat 9: 453-462, 2010.

10. Unger F, Dominikus K and Haselsberger K: Stereotactic radiosurgery and fractionated stereotactic radiotherapy of acoustic neuromas. HNO 59: 31-37, 2011 (In German).

11. Alongi F and Di Muzio N: Image-guided radiation therapy: A new era for the radiation oncologist? Int J Clin Oncol 14: 568-569, 2009.

12. Schweikard A, Shiomi H and Adler J: Respiration tracking in radiosurgery. Med Phys 31: 2738-2741, 2004.

13. Jereczed-Fossa BA, Beltramo G, Fariselli L, et al: Robotic image-guided stereotactic radiotherapy, for isolated recurrent primary, lymph node or metastatic prostate cancer. Int J Radiat Oncol Biol Phys 79: 1-9, 2011.

14. Reichner C, Collins B, Gagnon G, et al: The placement of gold fiducials for cyberknife stereotactic radiosurgery using a modified transbronchial needle aspiration technique. J Bronchol 12: 193-195, 2005.

15. Collins BT, Erickson K, Reichner CA, et al: Radical stereotactic radiosurgery with real-time tumor motion tracking in the treatment of small peripheral lung tumors. Radiat Oncol 2: 39, 2007.

16. Hashizume M: MRI-guided laparoscopic and robotic surgery for malignancies. Int J Clin Oncol 12: 94-98, 2007.

17. Roh KW, Jang JS, Kim MS, et al: Fractionated stereotactic radiotherapy as reirradiation for locally recurrent head and neck cancer. Int J Radiat Onc Biol Phys 74: 1348-1355, 2009.

18. King CR, Brooks JD, Gill H, et al: Stereotactic body radiotherapy for localized prostate cancer: interim results of a prospective phase II clinical trial. Int J Radiat Oncol Biol Phys 73: 1043-1048, 2009.

19. Brown WT, Fayad F, Hevezi J, et al: Individualized higher dose of 70-75 Gy using five-fraction robotic stereotactic radiotherapy for non-small-cell lung cancer: a feasibility study. Comput Aided Surg 16: 1-10, 2011.

20. Vavassori A, Jereczek-Fossa BA, Beltramo G, et al: Image-guided robotic radiosurgery as salvage therapy for locally recurrent prostate cancer after external beam irradiation: retrospective feasibility study on six cases. Tumori 96: 71-75, 2010.

21. Yoshioka Y: Current status and perspectives of brachytherapy for prostate cancer. Int J Clin Oncol 14: 31-36, 2009.

22. Thariat J, Li G, Angellier G, et al: Current indications and ongoing clinical trials with CyberKnife stereotactic radiotherapy in France in 2009. Bull Cancer 96: 853-864, 2009.

23. Xie Y, Djajaputra D, King CR, et al: Intrafractional motion of the prostate during hypofractionated radiotherapy. Int J Radiat Oncol Biol Phys 72: 236-246, 2008.

24. Fowler JF, Welsh JS, Howard SP, et al: Loss of biological effect in prolonged fraction delivery. Int J Radiat Oncol Biol Phys 159: 242-249, 2004.

25. Fuller DB, Naitoh J, Lee C, et al: Virtual HDR CyberKnife treatment for localized prostatic carcinoma: dosimetry comparison with HDR brachytherapy and preliminary clinical observations. Int J Radiat Oncol Biol Phys 70: 1588-1597, 2008.

26. Naito S: Recent developments and perspectives of image-guided precision surgery for malignant. Int J Clin Oncol 12: 69-70, 2007.

27. Hara W, Loo BW, Goffinet DR, et al: Excellent local control with stereotactic radiotherapy boost after external beam radiotherapy in patients with nasopharyngeal carcinoma. Int J Radiat Oncol Biol Phys 71: 393-400, 2008. 